\title{
Evaluation of a porosity method for wet calcium
}

\section{phosphate cements}

Ingrid Ajaxon ${ }^{1}$, Yassine Maazouz ${ }^{2}$, Maria-Pau Ginebra ${ }^{2}$, Caroline Öhman ${ }^{1}$ and Cecilia Persson ${ }^{1}$

${ }^{1}$ Division of Applied Materials Science, Department of Engineering Sciences, Uppsala University, Uppsala, Sweden

${ }^{2}$ Biomaterials, Biomechanics and Tissue Engineering Group, Department of Materials Science and Metallurgy, Technical University of Catalonia (UPC), Barcelona, Spain

\section{Corresponding author:}

Cecilia Persson, Division of Applied Materials Science, Department of Engineering Sciences, Uppsala University, The Ångström Laboratory, Box 534, SE-751 21 Uppsala, Sweden.

E-mail: cecilia.persson@angstrom.uu.se 


\section{Abstract}

The porosity of a calcium phosphate cement is a key parameter as it affects several important properties of the cement. However, a successful, nondestructive porosity measurement method that does not include drying has not yet been reported for calcium phosphate cements. The aim of this study was to evaluate isopropanol solvent exchange as such a method. Two different types of calcium phosphate cements were used, one basic (hydroxyapatite) and one acidic (brushite). The cements were allowed to set in an aqueous environment and then immersed in isopropanol and stored under three different conditions: at room temperature, at room temperature under vacuum $(300 \mathrm{mbar})$ or at $37^{\circ} \mathrm{C}$. The specimen mass was monitored regularly. Solvent exchange took much longer time to reach steady state in hydroxyapatite cements compared to brushite cements, 350 and $18 \mathrm{~h}$, respectively. Furthermore, the immersion affected the quasi-static compressive strength of the hydroxyapatite cements. However, the strength and phase composition of the brushite cements were not affected by isopropanol immersion, suggesting that isopropanol solvent exchange can be used for brushite calcium phosphate cements. The main advantages with this method are that it is non-destructive, fast, easy and the porosity can be evaluated while the cements remain wet, allowing for further analysis on the same specimen.

\section{Keywords}

Calcium phosphate, bone cement, porosity, solvent exchange, brushite, hydroxyapatite 


\section{Introduction}

For biomaterials based on injectable ceramic cements, porosity is a defining parameter that is often studied. The porosity and the pore size distribution influence important physical properties of the cement, such as the mechanical properties and the surface area. The mechanical properties determine what loads the material can sustain in vivo, and will therefore strongly affect the applications the material can be used for. However, strength is inversely proportional to the porosity, which in turn is proportional to the surface area, which affects the rate at which reactions with the material will occur, such as the rate of dissolution of the material in vivo. Furthermore, interconnected pores may facilitate vascularization and bone ingrowth.1,2

There are many different techniques for acquiring the porosity of a ceramic material. In the case of calcium phosphate cements (CPCs), commonly employed methods include helium pycnometry, ${ }^{3,4}$ mercury intrusion porosimetry, ${ }^{5-7}$ calculations based on tabulated material densities, ${ }^{5}$ scanning electron microscopy and microtomographic imaging5,8. Solvent resaturation ${ }^{9}$ has also been employed, and more recently, water evaporation was found to be a useful method to assess the porosity of $\mathrm{CPCs}^{7}$. However, there are disadvantages associated with all of the above-mentioned methods. In the case of mercury intrusion porosimetry, the equipment has a high running cost and a relatively long analysis time (approximately 3 hours), which can result in fewer replicates being analysed. The gas pycnometer used for helium pycnometry is also associated to a cost, and only one specimen can be measured at a time. Water evaporation and solvent resaturation do not have these drawbacks. However, all 
of these methods are destructive to a certain degree in terms of alteration of the cement's physical properties: in the case of mercury intrusion porosimetry the specimens are contaminated with mercury and cannot be used for further characterization; and for helium pycnometry, solvent resaturation and water evaporation the specimens are dried during analysis. Since the drying process can lead to an unwanted phase transformation in the cements ${ }^{7}$ and dry mechanical properties of CPCs are known to be greater than their wet counterpart, ${ }^{10-12}$ the same specimens used in porosity measurements cannot be used in subsequent experiments, where their properties in the wet condition are of interest.

Injectable CPCs consist of a powder and a liquid phase that are mixed to form a paste, which is then injected and allowed to set in vivo. When the injected cement paste has set it will be in direct contact with body fluids, which means that the material never dries. To mimic the in vivo situation more closely, it would therefore be advantageous to keep the CPCs wet when characterizing the mechanical properties and the porosity of the same specimens. However, as explained above, a non-destructive porosity measurement method, which does not include drying, is still lacking in the literature for self-setting CPCs.

Solvent exchange is a method commonly used for other types of cements, such as Portland cements and concrete. ${ }^{13}$ This method assumes that the volume of water within the wet cement is equal to the volume of the pores. First, the apparent volume, i.e. the volume that is described by the outer dimensions of the specimen, is determined using either a caliper or by Archimedes' principle; 
second, the initial mass of the wet specimen is recorded; and finally, the specimen is immersed in a solvent (normally an alcohol such as methanol or isopropanol) and the mass of the specimen is recorded until complete exchange occurs, i.e. when the solvent exchange process reaches steady-state. ${ }^{13}$ As the solvent through counter diffusion replaces the water, the mass of the specimen changes since the infiltrating solvent has a different density than the displaced water. The volume of the pores can then be calculated from the difference between the initial mass of the specimen and the mass after complete exchange, taking into account the densities of water and solvent. The ratio between the volume of the pores and the apparent volume of the specimen gives the porosity. Similarly to the water evaporation method, ${ }^{7}$ the only requirements for the solvent exchange method are a balance and a method to determine the apparent volume of the specimen; hence it is an easy method that can be employed in almost every laboratory. The biggest difference between the two methods is that solvent exchange can be performed while keeping the cements wet.

Several different organic liquids, such as methanol, ethanol, propan-1-ol and propan-2-ol, have been suggested for this method, for other types of cements. ${ }^{13}$ Solvent exchange using methanol as a solvent has previously been evaluated as a porosity measurement method for brushite CPCs, but methanol was found to affect the phase composition of the cement and was thus discarded as a useful method. ${ }^{7}$ It can be hypothesized that isopropanol, having a larger, less polar molecule compared to methanol would have less influence on the chemistry of the cement, ${ }^{7}$ and it was hence chosen as solvent in the current study. Isopropanol has also previously been successfully used for porosity 
measurements of $\mathrm{CPCs},{ }^{9}$ but in a solvent resaturation method, in which a dry specimen is soaked in the solvent until complete saturation. If the wet cement properties were desired, this method would hence also be inappropriate.

The aim of this study was to evaluate solvent exchange as a method to measure the porosity of wet CPCs, using isopropanol as a solvent. Such a method would allow for the porosity and, e.g. mechanical properties to be measured on the very same, wet cement specimen.

\section{Materials and Methods}

\section{Cement preparation}

Two different types of CPCs were used in this study, one basic (hydroxyapatite, HA) and one acidic (dicalcium phosphate dihydrate or brushite). The powder phase for the HA cement consisted of alpha-tricalcium phosphate ( $\alpha$-TCP), the preparation of which has been described elsewhere ${ }^{6}$ Briefly, calcium hydrogen phosphate (Sigma-Aldrich, St. Louis, MO, USA) and calcium carbonate (SigmaAldrich, St. Louis, MO, USA) were mixed in appropriate amounts, heated at $1400{ }^{\circ} \mathrm{C}$ for $15 \mathrm{~h}$ followed by quenching in air and then milled to a coarse powder (median particle size $5.2 \mu \mathrm{m}$ ). Precipitated HA (2 wt\%; Alco, Akron, OH, USA) was added as a nucleation agent in the powder, to increase the rate of formation of HA crystallites. The HA cement was prepared by mixing $\alpha$-TCP with a solution of 2.5 wt\% sodium hydrogen phosphate (Sigma-Aldrich, St. Louis, MO, USA), added to accelerate the setting reaction ${ }^{6}$. A liquid to powder (L/P) ratio of $0.35 \mathrm{ml} / \mathrm{g}$ was used and the powder and liquid phases were mixed in a mechanical mixing device (Cap-Vibrator, Ivoclar Vivadent AG, Schaan, 
Liechtenstein) for 1 minute. This composition of the basic CPC was chosen because its properties have been thoroughly studied before, in particular compressive strength and the cement porosity, by means of mercury intrusion. ${ }^{6,14}$ Cylindrical HA specimens (6 $\mathrm{mm}$ in diameter, $13 \mathrm{~mm}$ in height) were prepared and kept at room temperature (RT; $21 \pm 1^{\circ} \mathrm{C}$ ) for 60 minutes, to allow enough time for cement cohesion, before being immersed into approximately $40 \mathrm{ml}$ of phosphate buffered saline (PBS; Sigma-Aldrich, St. Louis, MO, USA; containing $0.01 \mathrm{M}$ phosphate buffer, $0.0027 \mathrm{M}$ potassium chloride and $0.137 \mathrm{M}$ sodium chloride, $\mathrm{pH}$ 7.4). The HA cement specimens were allowed to set in PBS for 7 days at $37^{\circ} \mathrm{C}$, to achieve complete setting, 6 .

For the brushite cements, as-received monocalcium phosphate monohydrate (MCPM; Scharlau, Sentmenat, Spain) was sieved, and only particle sizes below 75 $\mu \mathrm{m}$ were used. The powder phase for these specimens was then prepared by thoroughly mixing 45 mol\% MCPM $(<75 \mu \mathrm{m}), 55$ mol\% beta-tricalcium phosphate (ß-TCP; Sigma-Aldrich, St. Louis, MO, USA), together with 1 wt $\%$ disodium dihydrogen pyrophosphate (Sigma-Aldrich, St. Louis, MO, USA), acting as a retardant ${ }^{2}$. The as-received $ß-\mathrm{TCP}$ contains approximately 8-10 wt $\%$ betacalcium pyrophosphate (ß-CPP). The liquid phase was prepared by dissolving citric acid (Sigma-Aldrich, St. Louis, MO, USA) in double distilled water to a final concentration of $0.5 \mathrm{M}$. The powder and liquid phases were mixed in a CapVibrator for 1 minute, at an L/P-ratio of $0.22 \mathrm{ml} / \mathrm{g}$. The same composition of the brushite cement has previously been studied in terms of the mechanical properties, under compression and indirect tension, and the porosity, using helium pycnometry measurements. ${ }^{16}$ The cement paste was moulded into 
cylindrical moulds (6 $\mathrm{mm}$ in diameter, $13 \mathrm{~mm}$ in height) and the specimens were allowed to set for 5 minutes at RT. Each specimen was then immersed into $40 \mathrm{ml}$ PBS, and left to set at $37^{\circ} \mathrm{C}$ for 24 hours $^{16}$.

Set specimens were polished plane parallel using SiC paper to a final height of 12 mm (sample dimensions according to ASTM F451 standard ${ }^{17}$ ).

\section{Solvent exchange}

To determine the apparent volume, $V_{a}$, of all set specimens, a density kit (Mettler Toledo, Greifensee, Switzerland) based on Archimedes' principle was used. The surfaces of each specimen were dried with a moist tissue paper and the initial mass of the wet specimen was measured. The specimen was then immersed in water and the mass of the specimen in water was recorded. $V_{a}$ was calculated using equation (1):

$$
V_{a}=\frac{m_{a i r}-m_{H_{2} O} O}{\rho_{H_{2} O}}
$$

where $m_{\text {air }}$ is the mass of the wet specimen in air, $m_{\mathrm{H}_{2} \mathrm{O}}$ is the mass of the wet specimen in water and $\rho_{\mathrm{H}_{2} \mathrm{O}}$ is the density of water (approximately $1 \mathrm{~g} / \mathrm{cm}^{3}$ at RT).

Each wet specimen was then immersed in $10 \mathrm{ml}$ (approximately 100 times the volume of the water in the pores ${ }^{7}$ ) of isopropanol (Merck KGaA, Darmstadt, Germany) and kept under three different storage conditions: (1) RT, (2) RT under vacuum (300 mbar) or (3) at $37^{\circ} \mathrm{C}$ (20 specimens for each group). Small amounts of isopropanol were added during the study to account for evaporation and maintain a constant volume. Storage condition 2 was chosen under the 
hypothesis that the exchange process would go faster under lower pressure. Storage condition 3 was chosen since the CPCs used in this study are supposed to be injected and set under physiological conditions, and it was hypothesized that the higher temperature could have an effect on the rate of exchange and/or phase composition of the resulting material. The mass of each specimen was monitored regularly until complete solvent exchange, which was deemed to occur when the mass change had reached steady-state. The precision of the mass measurement was $0.017 \mathrm{~g}$, hence, to be able to resolve actual mass loss from uncertainties in the mass measurements, an accuracy of $0.017 \mathrm{~g}$ for two consecutive time points was used to determine whether steady-state was reached or not. After steady-state had been reached, all specimens were kept in isopropanol for twice the amount of time to allow time for complete exchange for all groups, i.e. approximately 700 hours and 48 hours for HA and brushite, respectively. The total porosity, $\Phi_{\text {solv.ex, }}$ of the specimen was then calculated using equation (2):

$$
\Phi_{\text {solv.ex. }}(\%)=\frac{\left(m_{\text {air }}-m_{\text {solvent }}\right) /\left(\rho_{\mathrm{H}_{2} \mathrm{O}}-\rho_{\text {solvent }}\right)}{V_{a}} \times 100 \text {, }
$$

where $m_{\text {solvent }}$ is the mass of the specimen after complete exchange, and $\rho_{\text {solvent }}$ is the density of the solvent used, in this case isopropanol having a density of $0.786 \mathrm{~g} / \mathrm{cm}^{3}$ at RT. $\Phi_{\text {solv.ex. }}$ will hereafter be referred to as solvent exchange porosity.

\section{Mechanical testing}

After complete solvent exchange was reached for all groups (700 hours and 48 hours for HA and brushite, respectively), the specimens were tested in quasistatic compression under ambient conditions using a materials testing machine 
(AGS-X, Shimadzu, Kyoto, Japan), equipped with a $5 \mathrm{kN}$ load cell, at a displacement rate of $1 \mathrm{~mm} / \mathrm{min}$. The compressive strengths of HA and brushite (20 replicates of each type of cement) that had set in PBS at $37^{\circ} \mathrm{C}$ for 7 days and 24 hours, respectively, served as controls. All specimens were kept wet (control group soaked in PBS, all other specimens in isopropanol) up until the moment of quasi- static compression of each respective specimen.

\section{Phase characterisation}

Immediately after the specimens were tested mechanically, they were ground into a fine powder and analysed with X-ray diffraction (XRD; D8 Advance, Bruker AXS GmbH, Karlsruhe, Germany) using a theta-theta setup with Ni-filtered $\mathrm{Cu}-\mathrm{K} \alpha$ irradiation. For each group, the ground powder from all specimens was homogenized into a single quantity of material and six specimens were taken at random from this quantity to have enough material for XRD and Rietveld analysis. Diffraction patterns were collected between $2 \theta$ of 10 to 60 degrees, in steps of 0.02 and $0.25 \mathrm{~s}$ per step, with a sample rotation speed of $80 \mathrm{rpm}$ and using a beam knife. Quantitative phase composition analysis was done by Rietveld refinement using the BGMN software (www.bgmn.de) ${ }^{18}$ with Profex (http://profex.doebelin.org) as user interface. The reported result was the mean of six independent measurements with the repeatability taken as $2.77 \mathrm{x}$ standard deviation according to ASTM E177-14. ${ }^{19,20}$ Crystalline models were taken from PDF\# 01-074-056521 for HA and PDF\# 04-010-434822 for $\alpha$-TCP, PDF\# 04-008$8714^{23}$ for ß-TCP, PDF\# 04-013-334424 for brushite, PDF\# 04-009-3876 25 for ßCPP, and PDF\# 04-009-375526 for monetite. No other phases were identified in the diffraction patterns. 


\section{Comparison of methods}

Solvent exchange was validated as a porosity measurement method for brushite cements against the porosity obtained from water evaporation and helium pycnometry. The decision to use only brushite specimens for validation was based on the results. The same cement composition and preparation method as above was used. A total of 40 specimens were used. After the cements had set for 24 hours in $\mathrm{PBS}$ at $37^{\circ} \mathrm{C}$, the apparent volume, $V_{a}$, and density, $\rho_{a}$, were determined using Archimedes' principle, as previously described. The specimens were then divided into two groups, with 20 validation specimens in each group. The porosity of the specimens in the first group was evaluated by solvent exchange and the specimens in the second group by water evaporation ${ }^{7}$ followed by helium pycnometry. The porosity of the 20 specimens in the first group was calculated using equation (2) after they had been immersed in isopropanol (10 ml/specimen) at RT for 18 hours. The 20 specimens in the second group were dried under vacuum (300 mbar) at RT for 24 hours and the volume of the evaporated water, $V_{w}$, was determined by weighing each specimen again. The porosity, $\Phi_{\mathrm{H}_{2} \text { Oevap. }}$, of the specimens was calculated using equation (3)7:

$$
\Phi_{H_{2} \text { Oevap. }}(\%)=\left(\frac{V_{w}}{V_{a}}\right) \times 100 \text {. }
$$

$\Phi_{\mathrm{H}_{2} \text { Oevap. }}$ will hereafter be referred to as water evaporation porosity.

Then, the same 20 dried specimens were crushed and homogenized into a single quantity of material, from which six samples were taken at random and the skeletal densities, $\rho_{s}$, were determined using a helium pycnometer (AccuPyc 1340, Micromeritics, Norcross, GA, USA; maximum pressure of 19.5 Psi, chamber 
size of $1 \mathrm{~cm}^{3}$ ) using 20 purges and 10 runs. The porosity, $\Phi_{H e}$, of the specimens was calculated using equation (4)

$$
\Phi_{H e}(\%)=\left(1-\frac{\rho_{a}}{\rho_{s}}\right) \times 100 \text {, }
$$

where $\rho_{a}$ is the apparent density determined by Archimedes' principle. $\Phi_{H e}$ will hereafter be referred to as helium pycnometry porosity.

\section{Correlation between quasi-static compressive strength and porosity}

The relationship between porosity, as evaluated by isopropanol exchange at RT, and the quasi-static compressive strength for each brushite cement specimen was investigated and the compressive strength of a fully dense cement (zero porosity), $\sigma_{C 0}$, was calculated using equation $(5)^{27}$ :

$$
\sigma_{C}=\sigma_{C 0} e^{-q \Phi}
$$

where $\sigma_{C}$ is the compressive strength, $\Phi$ the porosity and $q$ is a dimensionless constant. The curve fitting was done in the Curve Fitting Toolbox ${ }^{\mathrm{TM}}$ of $_{\text {MATLAB }}{ }^{\circledR}$ (version R2012a, The MathWorks ${ }^{\circledR}$ Inc., Natick, MA, USA).

\section{Statistical analysis}

IBM $^{\circledR}$ SPSS $^{\circledR}$ Statistics (Version 19, IBM Corp., Armonk, NY, USA) was used for the statistical analysis. Analysis of variance (ANOVA) was used to compare compressive strengths at a significance level of $\alpha=0.05$. For HA cements, Tamhane's post hoc test was used since Levene's test did not confirm homogeneity of variances. Scheffe's post hoc test was used for brushite cements. Chauvenet's criterion was applied once to discard outliers. A t-test was used to compare porosity values obtained from water evaporation and helium 
pycnometry measurements with those from solvent exchange at a significance level of $\alpha=0.05$.

\section{Results}

\section{Solvent exchange}

HA specimens kept in isopropanol at RT or at RT under vacuum reached steadystate after approximately 350 hours, see Figure 1 . The average mass loss was $4.5 \pm 0.1 \%$ and $4.3 \pm 0.2 \%$ for specimens kept at RT or RT under vacuum, respectively. The specimens kept in isopropanol at $37^{\circ} \mathrm{C}$ lost mass faster than specimens kept at RT or under vacuum at RT, and steady-state was reached after approximately 300 hours, with an average mass loss of $5.0 \pm 0.1 \%$. When steadystate had been reached, the porosity was calculated (equation (2)) and for specimens kept in isopropanol at RT the average porosity was $43.9 \pm 1.2 \%$; for specimens kept under vacuum at RT it was $42.4 \pm 1.7 \%$; and for specimens kept at $37^{\circ} \mathrm{C}$ it was $48.5 \pm 1.3 \%$. After approximately 700 hours immersion time in isopropanol the average mass loss for the three groups were: $4.8 \pm 0.1 \%$ (RT); $4.8 \pm 0.2 \%$ (RT under vacuum); and $5.1 \pm 0.1 \%\left(37^{\circ} \mathrm{C}\right)$. 


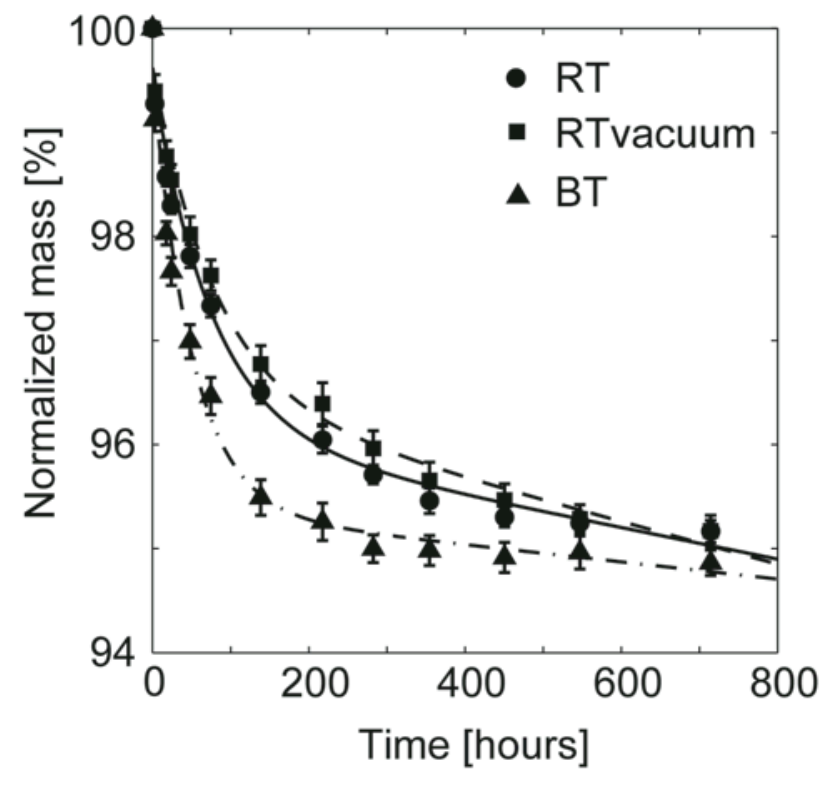

Figure 1. Mass change of HA cements kept in isopropanol under different storage conditions, $\mathrm{n}=20$.

For brushite specimens kept in isopropanol at RT the mass loss reached steadystate after approximately 18 hours, see Figure 2. The behaviour of specimens kept at RT under vacuum followed a similar mass loss pattern as those kept at RT, and steady-state was also reached after 18 hours of immersion. The average mass loss for brushite specimens was approximately $1.3 \pm 0.2 \%$ and $1.0 \pm 0.1 \%$ when steady-state conditions were reached for specimens kept at RT and at RT under vacuum, respectively. When complete solvent exchange was reached, the average porosity (equation (2)) for specimens kept in isopropanol at RT was $12.5 \pm 1.6 \%$ and, $10.4 \pm 1.2 \%$ for specimens kept at RT under vacuum. After 48 hours of immersion in isopropanol, the average mass loss was $1.3 \pm 0.2 \%$ (RT) and $1.2 \pm 0.1 \%$ (RT under vacuum). At $37^{\circ} \mathrm{C}$, the specimens experienced a steady decrease in mass over time and never reached steady-state (using an accuracy of 0.017g for two consecutive time points as described above) during the period of measurement, thus the porosity could not be determined. 


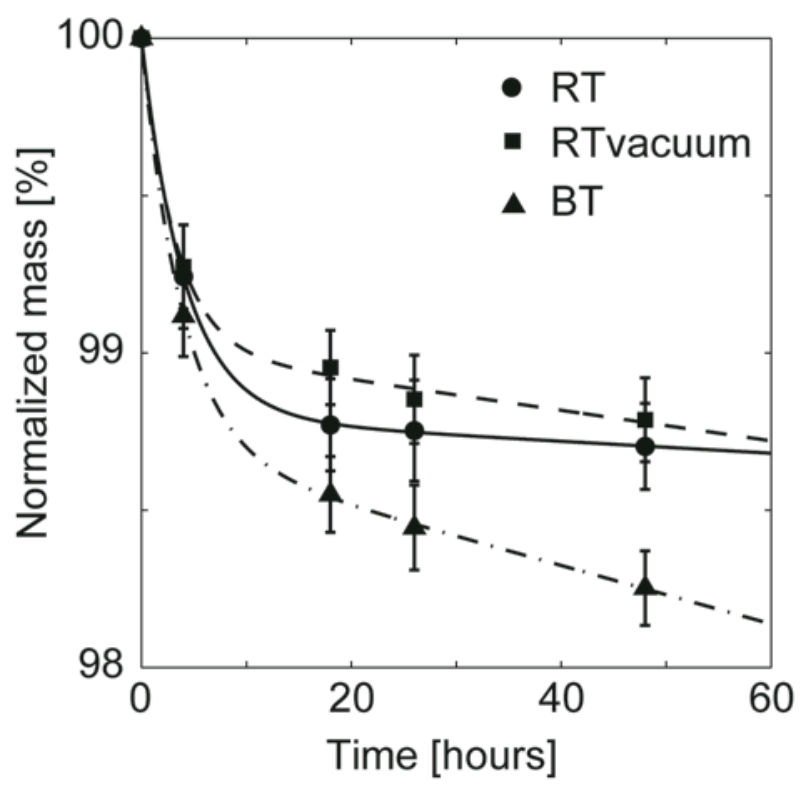

Figure 2. Mass change of brushite cements kept in isopropanol under different storage conditions, $\mathrm{n}=20$.

\section{Mechanical testing}

The quasi-static compressive strengths of the HA cements are shown in Figure 3. The control group had a strength of $40.7 \pm 7.2 \mathrm{MPa}$, the strength of the other groups were: $56.8 \pm 14.0 \mathrm{MPa}$ for specimens kept at RT; $52.9 \pm 8.5 \mathrm{MPa}$ for the group kept at RT under vacuum and $55.0 \pm 13.4 \mathrm{MPa}$ for those kept at $37^{\circ} \mathrm{C}$. There was a significant difference $(\mathrm{p} \leq 0.001)$ in strength between the control group and the three other groups, whereas when comparing RT, RT vacuum and $37^{\circ} \mathrm{C}$ no significant difference $(\mathrm{p} \geq 0.088)$ in strength was found. 


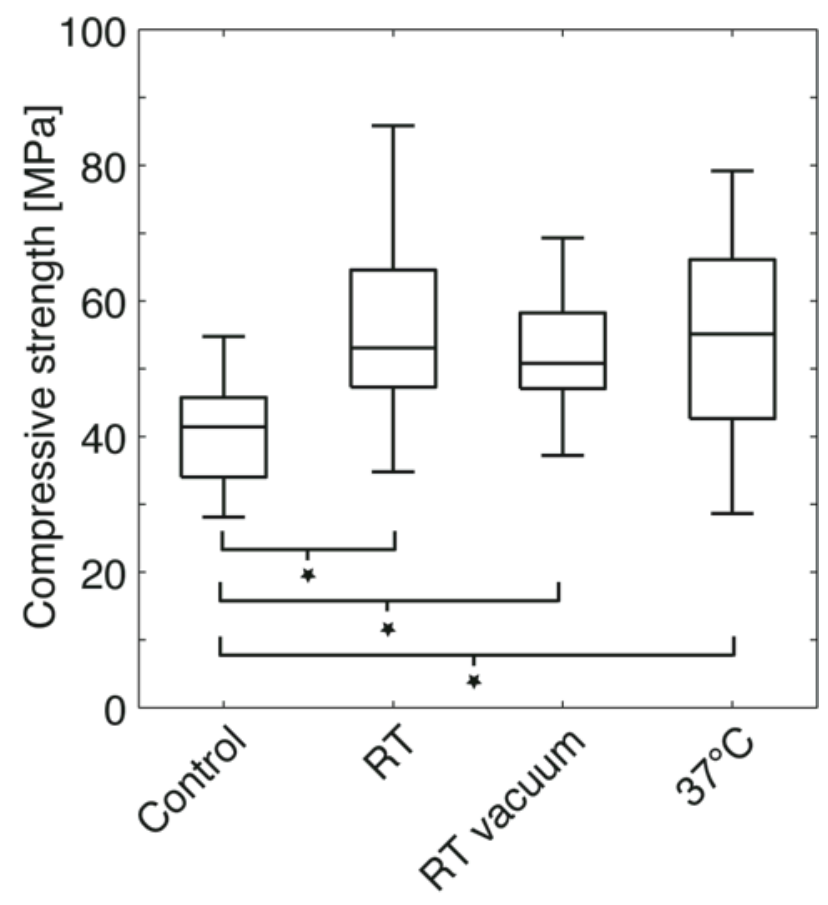

Figure 3. Quasi-static compressive strength of HA cements, $n=20 /$ group. *Statistically significant difference between groups $(\alpha=0.05)$.

In Figure 4, the results from the mechanical testing of brushite cements are shown. The quasi-static compressive strength was 55.1 $\pm 10.2 \mathrm{MPa}$ for the control group; $61.2 \pm 11.2 \mathrm{MPa}$; $58.1 \pm 15.8 \mathrm{MPa}$; and $57.7 \pm 12.6 \mathrm{MPa}$ for specimens kept at RT, RT under vacuum and $37^{\circ} \mathrm{C}$, respectively. The differences in strength between the four groups were not statistically significant $(p=0.26)$. 


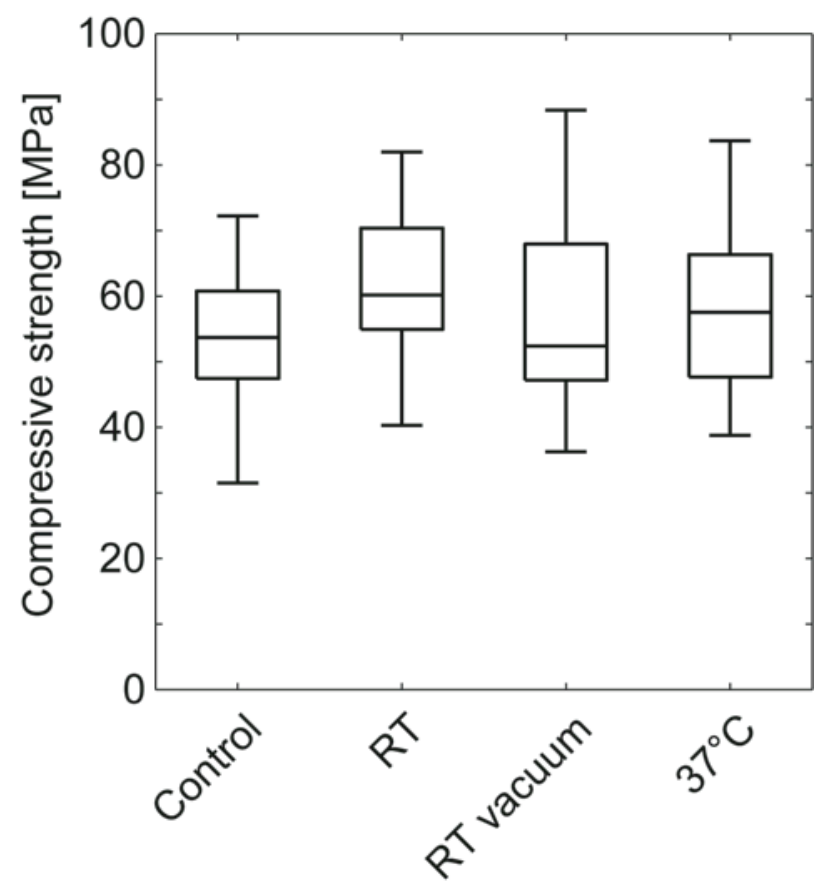

Figure 4. Quasi-static compressive strength of brushite cements, n=20/group. No statistically significant differences were found between groups $(\alpha=0.05)$.

\section{Phase characterisation}

HA specimens in the control group contained 92 wt $\%$ HA, 6 wt $\% \alpha$-TCP and $2 \mathrm{wt} \%$ ß-TCP (with a repeatability better than $0.5 \mathrm{wt} \%$ ). In Figure 5, the results from the Rietveld refinement of the XRD measurements can be seen. The amounts of the crystalline phases did not change when the specimens were kept under different storage conditions. 


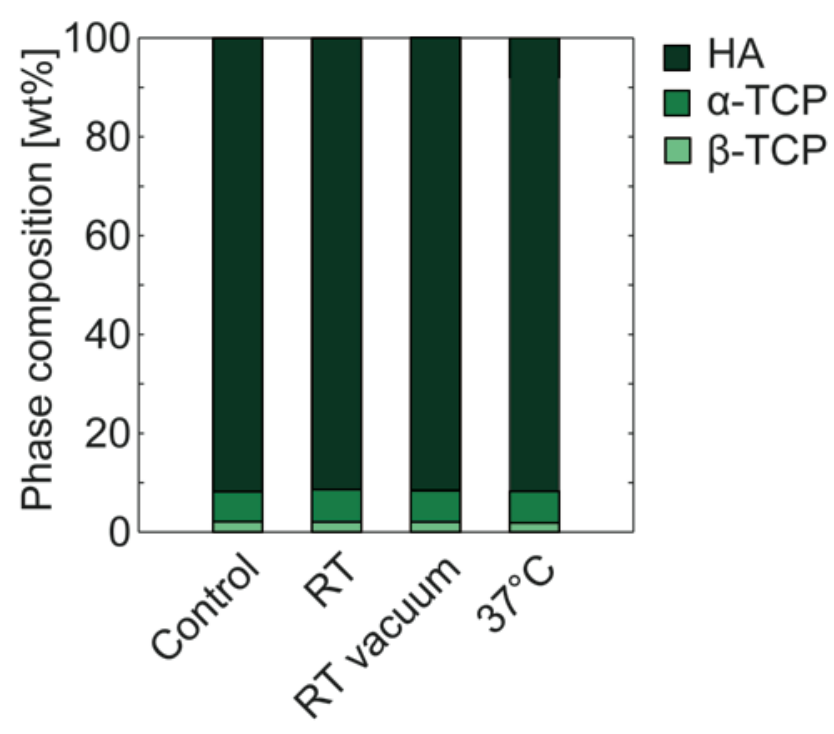

Figure 5. Phase composition of HA cements, n=6/group. The repeatability was better than $0.5 \mathrm{wt} \%$ for all groups.

XRD patterns of HA cements from the four groups (one representative pattern from each group) are shown together with reference patterns in Figure 6(a). The accuracy of the Rietveld calculations is shown in Figure 6(b), where observed and calculated peaks are shown together with the difference between the same.

(a)

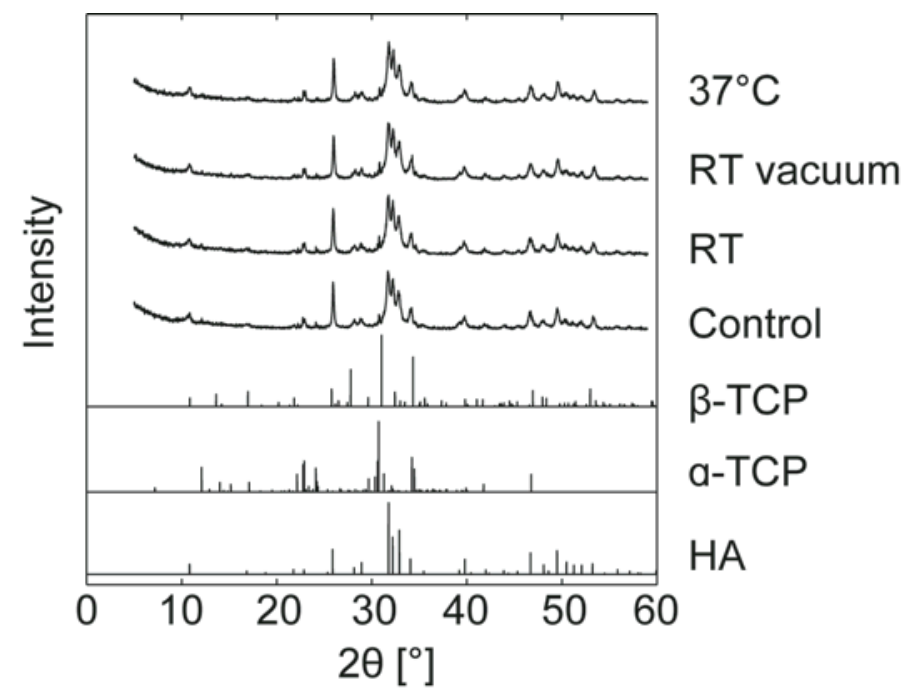




\section{(b)}

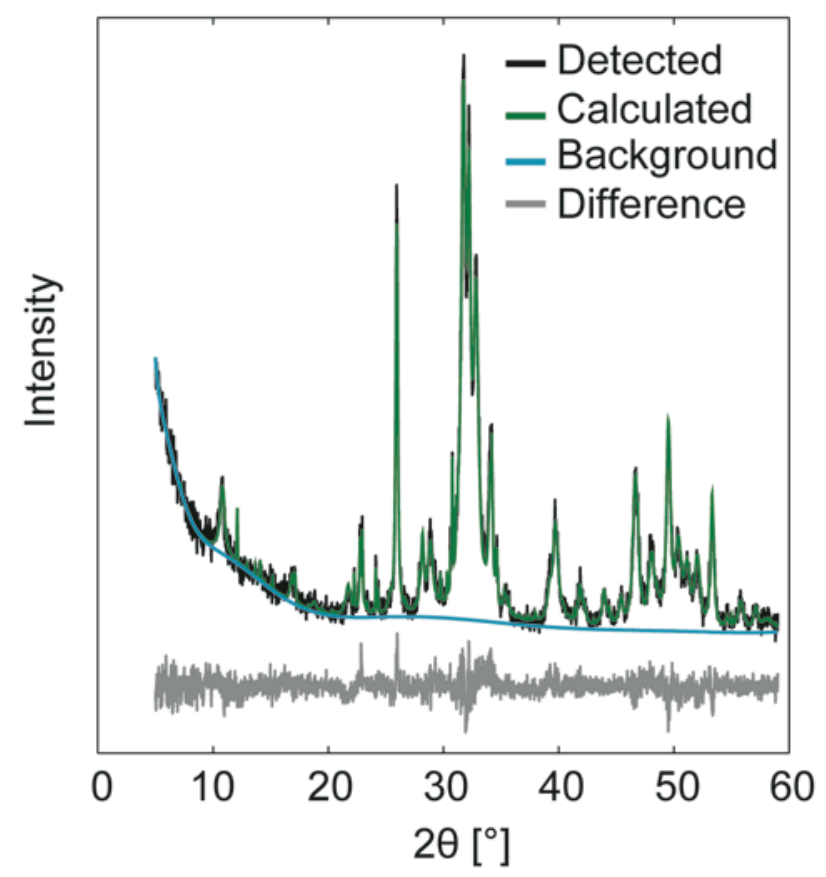

Figure 6. (a) XRD patterns for HA specimens kept under different storage conditions (one out of six measurements is shown). Reference patterns taken from PDF\# 01-074-0565 (HA), PDF\# 04-010-4348 ( $\alpha$-TCP) and PDF\# 04-0088714 (ß-TCP). (b) Representative XRD pattern from the BGMN software, showing the accuracy of the Rietveld refinement.

The brushite specimens from the control group contained 82 wt $\%$ brushite, $8 \mathrm{wt} \%$ ß-TCP, $6 \mathrm{wt} \%$ ß-CPP, and $4 \mathrm{wt} \%$ monetite (with a repeatability better than $1 \mathrm{wt} \%$ ). No MCPM could be detected. Results from the Rietveld refinement of XRD measurements of brushite cements (see Figure 7) showed that the amounts of the four crystalline phases present in the cements were comparable between the control group and the three other groups. 


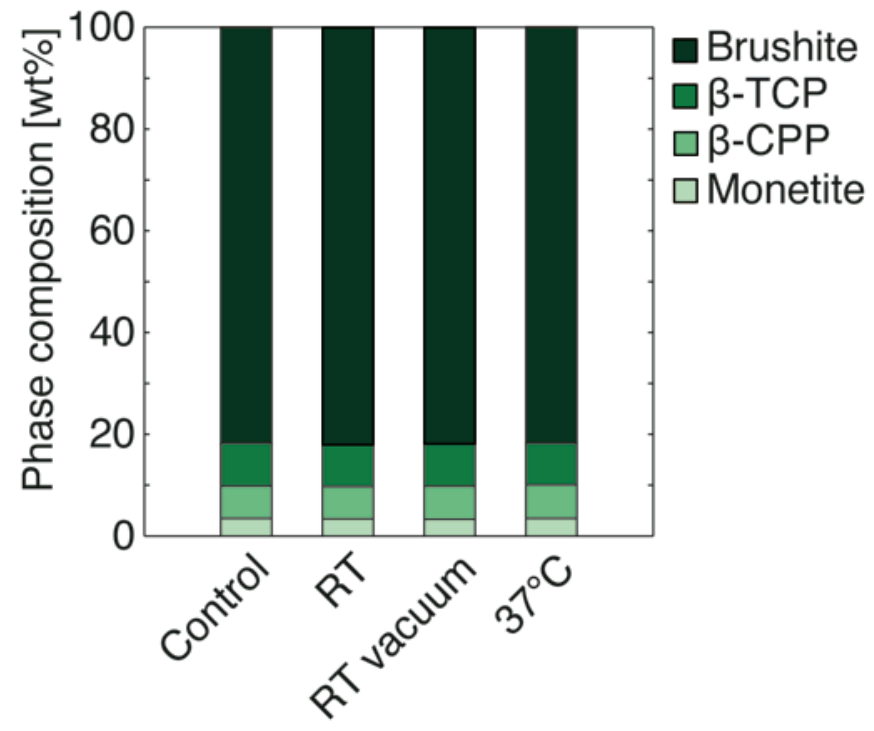

Figure 7. Phase composition of brushite cements, $n=6 /$ group. Relative error was lower than $1 \mathrm{wt} \%$ for all groups.

Reference patterns of monetite, ß-CPP, ß-TCP and brushite, can be seen in Figure 8(a), together with one representative pattern for each group. The accuracy of the Rietveld calculations is shown in Figure 8(b), where observed and calculated peaks are shown together with the difference between the same.

(a)

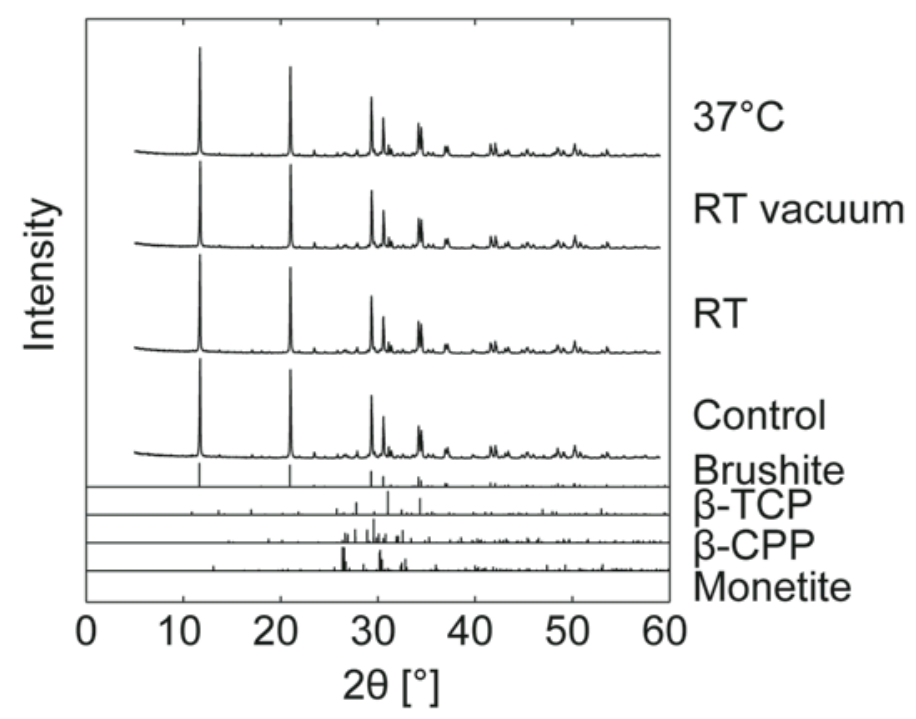


(b)

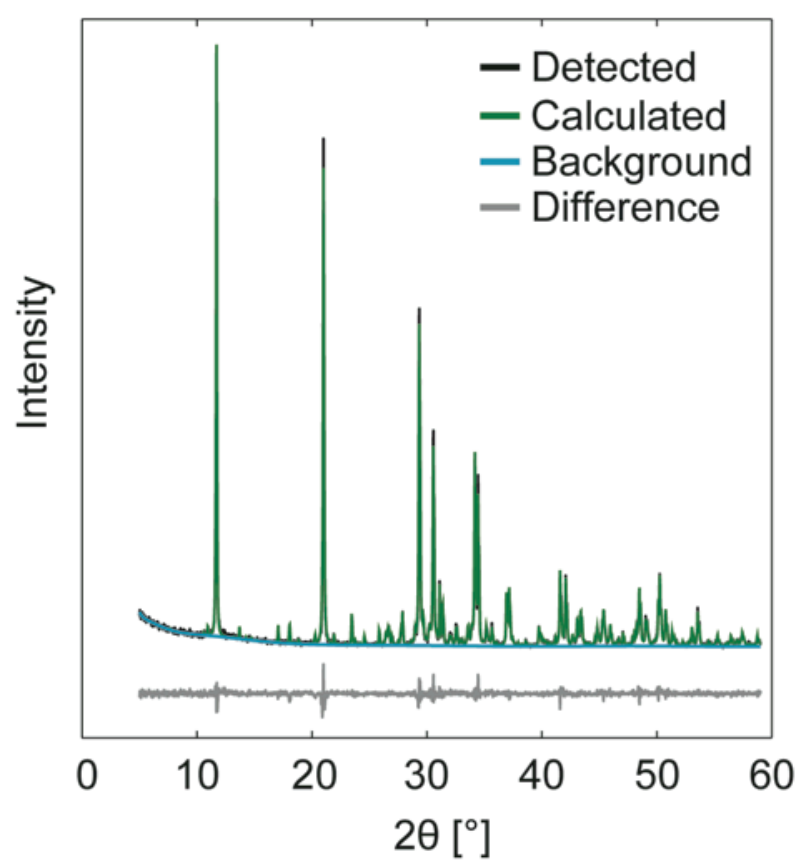

Figure 8. (a) XRD patterns for brushite specimens kept under different storage conditions (one out of six measurements is shown). Reference patterns taken from PDF\# 04-009-3755 (monetite), PDF\# 04-009-3876 (ß-CPP), PDF\# 04-0088714 (ß-TCP) and PDF\# 04-013-3344 (brushite). (b) Representative XRD pattern from the BGMN software, showing the accuracy of the Rietveld refinement.

\section{Comparison of methods}

The result of the validation experiments can be seen in Figure 9. The porosity of the first group of validation cements, evaluated by solvent exchange, was slightly lower $(10.2 \pm 1.0 \%)$ compared to the porosity of the second group of validation cements $(11.7 \pm 1.3 \%$ and $11.7 \pm 1.1 \%$ for water evaporation and helium pycnometry, respectively). 


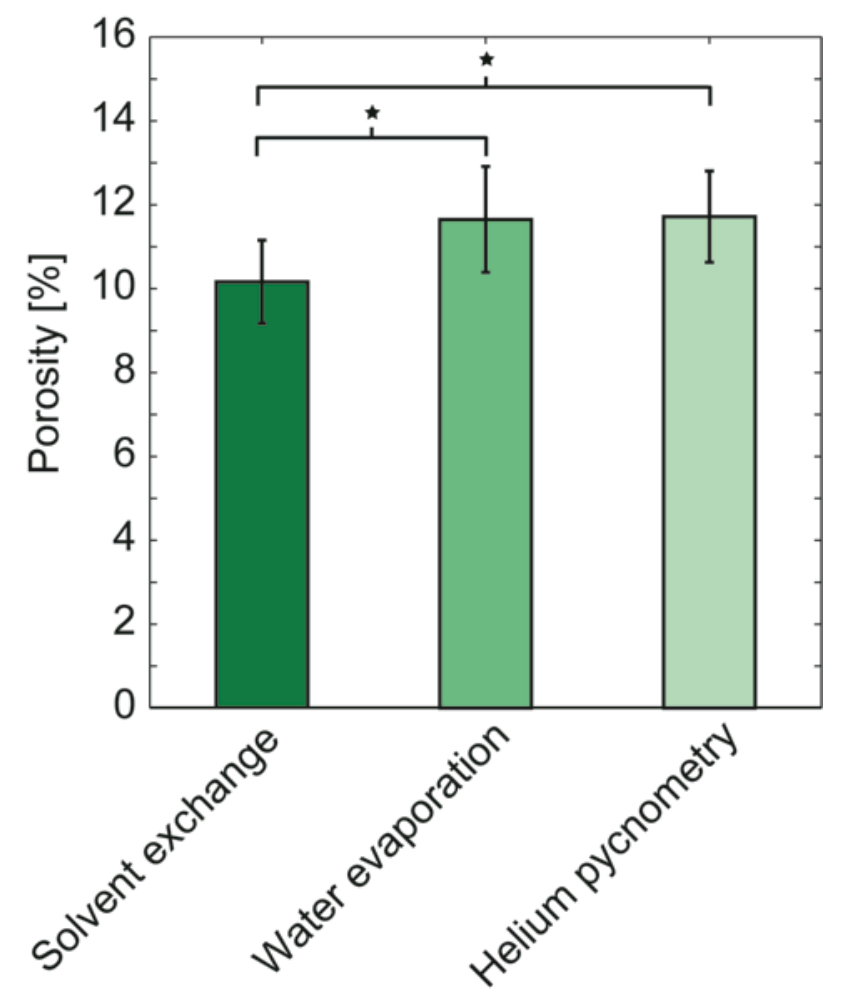

Figure 9. Porosity of brushite cements evaluated by either solvent exchange ( $n=20$, group 1) or water evaporation $(n=20$, group 2$)$ followed by helium pycnometry ( $\mathrm{n}=6$, group 2). *Statistically significant difference between groups $(\alpha=0.05)$.

The statistical analysis showed that the difference in porosity between solvent exchange and water evaporation was significant $(\mathrm{p}<0.001)$ and so was the difference in porosity between solvent exchange and helium pycnometry $(p=0.003)$. No significant difference could be seen between water evaporation and helium pycnometry $(\mathrm{p}=0.5)$.

\section{Correlation between quasi-static compressive strength and porosity}

The correlation between the porosity of brushite cements evaluated by solvent exchange (at RT) and the quasi-static compressive strength can be seen in Figure 
10. The strength of a fully dense cement, $\sigma_{C 0}=162 \pm 55 \mathrm{MPa}$, was calculated from the curve fit.

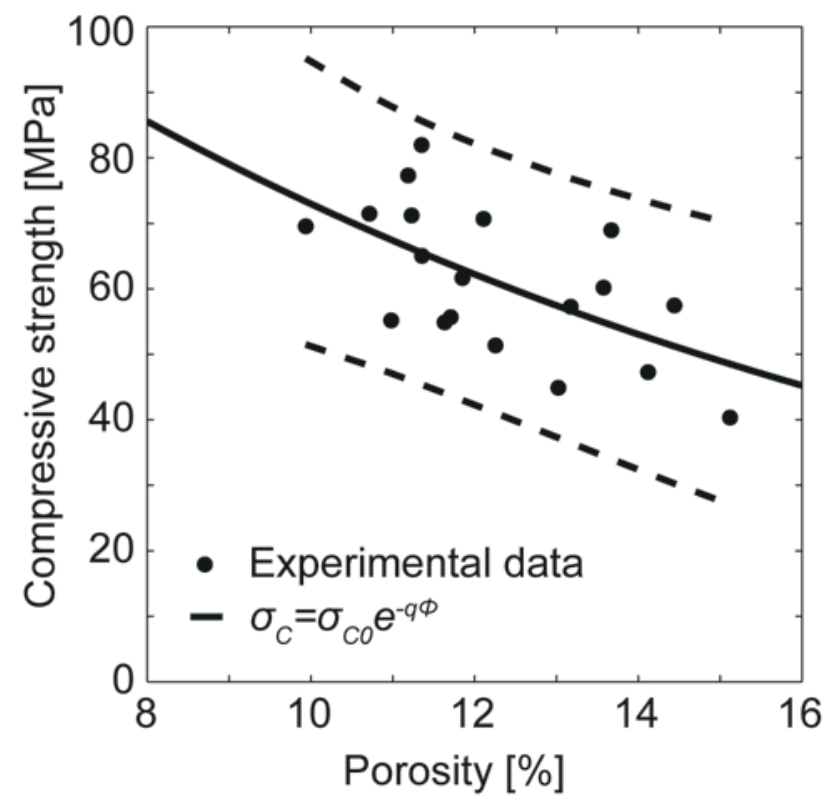

Figure 10. Porosity and strength values for brushite cement specimens kept at RT. Fit to equation (5) is shown as a continuous line and 95\% confidence bounds are shown as dotted lines.

\section{Discussion}

In this study, isopropanol was evaluated in a solvent exchange method in order to assess the porosity of CPCs while keeping them wet. It took approximately 350 hours for the solvent exchange to reach steady-state in HA specimens, compared to approximately 18 hours for brushite cements. This may be due to the smaller pore sizes and higher tortuosity in HA cements compared to brushite cements, which, consequently, retards the diffusion process. In fact, the pore size distribution for the same HA cement composition (although different preparation techniques - Espanol et al. and Canal et al.6,14 mixed their cement pastes by hand, herein cements pastes have been mixed mechanically) was found to range between 0.01 to $1 \mu \mathrm{m}$, with most of the pores lying around $0.01 \mu \mathrm{m}^{6,14}-$ 
which is one hundred times smaller than those found in the same brushite cement composition (although using an L/P-ratio of $0.3 \mathrm{ml} / \mathrm{g}$, which is higher compared to what was used herein) ${ }^{7}$. HA cements also had a higher total porosity compared to brushite cements, further contributing to the difference in exchange time; in this study the average porosity (at RT) was found to be around 43.9\% for HA cements (similar to what has previously been found for the same cement composition, $38 \pm 7 \%)^{14}$, compared to $12.5 \%$ for brushite cements. This porosity difference is related to the average mass loss for the two types of cements; the HA cements lost on average $4.8 \%$ in mass and the brushite cements $1.3 \%$ in mass after approximately 700 hours and 48 hours in isopropanol (at RT), respectively. Similar numbers in mass loss were seen for the other storage conditions.

The quasi-static compressive strength of the HA cements in the control group $(40.7 \pm 7.2 \mathrm{MPa})$ is in accordance with previous findings for a similar cement (approximately $42 \pm 11 \mathrm{MPa})^{6,14}$. Interestingly, the cements became stronger after being immersed in isopropanol for approximately 700 hours. It has been shown previously that the transformation of $\alpha$-TCP to HA can continue when allowing for longer setting times, ${ }^{15}$ and this could contribute to the increase in strength. However, the Rietveld refinement (Figures 5 and 6) suggested that the amount of unreacted $\alpha$-TCP in the cements did not change during the time of immersion, which contrasts this explanation. Previous studies have shown that mechanical properties are higher for dry cements compared to wet cements. ${ }^{10-12}$ During the long immersion time in isopropanol, the HA cements could have started to dehydrate, which could be another explanation for the increase in strength seen 
in this study. To explore the reasons further, any possible chemical reactions between the isopropanol and the HA cements should be investigated. In addition, a more thorough Rietveld refinement strategy, not only taking phase compositions but also changes in crystallite sizes into account, could be of interest.

For the brushite specimens that were kept in isopropanol at RT or under vacuum at RT, the isopropanol exchange process reached steady-state after approximately 18 hours. There was only a minor change in mass loss $(0.06 \%)$ between 18 and 48 hours of immersion at RT, hence an immersion time between 18 and 48 hours is advocated by the authors. The porosity obtained in this study for brushite cement $(10.4-12.5 \%)$ is comparable to what has been found previously for the same cement composition using helium pycnometry $(13.4 \pm 0.7 \%)^{16}$. However, in that study the amount of unreacted $ß-T C P$ left in the cements after setting was slightly greater (11 wt\%) than what was found in the cements in this study (8 wt\%), which might explain the slightly higher porosity values for those cements. When the porosity of a similar brushite cement composition was investigated with methanol used as a solvent for the exchange method, the amount of monetite increased almost threefold, preventing methanol to be used for the solvent exchange method. ${ }^{7}$ In the current study, using isopropanol instead, neither the quasi-static compressive strength (Figure 4), nor the phase composition of the brushite specimens (Figure 7) was affected. Since no major differences were seen when storing the specimens at RT and at RT under vacuum, storage at RT is suggested for future use of the method due to its simplicity. The validation experiments showed that the porosity of the 
brushite CPCs appeared to be slightly underestimated ( $\sim 1.5$ percentage points) when the solvent exchange method was used, as seen from the t-tests comparing solvent exchange to water evaporation and helium pycnometry (a difference that was statistically significant). However, the porosity values obtained from water evaporation and helium pycnometry are for cements that have been dried, and the drying process could slightly increase the porosity of the cements, by e.g. a phase transformation to a denser phase (dehydration from brushite to monetite). Also, while the water evaporation method and helium pycnometry were applied to the same specimens, a different set of specimens was used for the solvent exchange method. In fact, the difference in porosity between the validation experiments ( 1.5 percentage points) is lower than the batch-tobatch porosity variation seen for brushite cements in this study $(\sim 2.3$ percentage points), suggesting that the significant difference between methods could rather be an effect of batch-to-batch variation.

When brushite specimens were kept in isopropanol at $37^{\circ} \mathrm{C}$, steady-state was never reached during the time frame of the measurements. After 48 hours in isopropanol, the specimens kept at $37^{\circ} \mathrm{C}$ had lost on average $1.7 \%$ of mass, and the trend was continuously decreasing, compared to specimens kept at RT or at RT under vacuum, which had already reached steady-state conditions and had an average mass loss of approximately $1.2-1.3 \%$ after 48 hours in isopropanol. A possible explanation could be that the brushite specimens kept at $37^{\circ} \mathrm{C}$ had started to transform into monetite, as these types of calcium phosphate cements are known to undergo a phase transformation at elevated temperatures ${ }^{7}$. However, there was no phase transformation evident from the XRD 
measurements and Rietveld refinement, possibly due to very small changes not detectable by these types of analyses.

When applying this method to other types of brushite cements, the amount of isopropanol and the immersion times might need to be adjusted in order to account for different specimen sizes, shapes and porosities. While cements with larger interconnected pores might entail that shorter times can be used, larger sized specimens might need longer times of immersion for complete exchange.

One limitation of the method is that the size of the isopropanol molecule $(\sim 9 \AA)$ prevents it from penetrating all pores that contain water (size of water molecule $\sim 3 \AA ̊)$, hence there will still be pores containing water when complete exchange has been reached. Another limitation is that only open pores will be accessible to the isopropanol molecule; closed pores will not be accounted for using the solvent exchange method. Even though this entails that the method may underestimate the total porosity of the cements, other methods have the same limitations. Hence, we believe that solvent exchange, using isopropanol as solvent, can be a useful method for brushite CPCs, whenever a fast, easy and nondestructive way of assessing the porosity of wet cements is needed. The method allows the user to analyse many replicates at the same time, giving it a strong position against other time-consuming and costly methods, and is especially useful when there is a need to characterise the mechanical properties and the porosity of the same cement specimens. And importantly, since injectable cements are never dried during the delivery process in the operating room, and will be in direct contact with body fluids when injected, solvent exchange, which 
allows keeping the cements wet during evaluation, permits to more closely mimic the in vivo situation.

The application of the method in terms of evaluating the wet strength on the same specimen whose porosity was evaluated, was exemplified for the brushite cements kept at RT (Figure 10). Even though no attempt of varying the porosity of the cement specimens was done in this study, the small variation in porosity from the fabrication was enough to distinguish a decreasing trend in compressive strength with increasing porosity. The strength of a cement with zero porosity (162 $\pm 55 \mathrm{MPa}$ ) could be estimated, but the uncertainty was rather large due to the limited range in porosity. A more precise value of the strength of a fully dense cement would require a dedicated study with a wider range of porosities. However, it is in the same order of magnitude as what has been previously found for similar cement compositions (252 $\mathrm{MPa})^{2,28}$. They prepared several different cement compositions in terms of molar ratio between MCPM and ß-TCP, L/P-ratio and citric acid concentration; and the value of $\sigma_{C 0}$ was calculated from a broad range of compositions. In contrast to the data that Unosson and Engstrand et al. presented, ${ }^{2,28}$ the correlation between strength and porosity presented herein is for each and every cement specimen. The possibility to correlate strength and porosity for each and every cement specimen demonstrates the strength of the solvent exchange method compared to other porosity measurement techniques. 


\section{Conclusions}

Solvent exchange, using isopropanol as organic solvent, was used as a method to assess the porosity of wet CPCs. It proved to be an easy and fast method to determine the porosity of wet brushite CPCs. However, the isopropanol exchange took much longer time to reach steady-state in HA cements compared to brushite cements. Furthermore, even though immersion in isopropanol did not change the phase composition of the HA cements, the strength increased, which indicates that it is not an appropriate method for such cements. Conversely, for brushite cements the solvent exchange process took 18 hours at RT, and it did neither affect the strength nor the phase composition of the cements. Furthermore, porosity values obtained were similar to those obtained with other established porosity measurement techniques, making it a fast, easy and nondestructive method to determine the porosity for such cements under wet conditions.

\section{Declaration of conflicting interest}

The authors declared no potential conflicts of interest with respect to the research, authorship, and/or publication of this article.

\section{Funding}

This work was supported by the Swedish Foundation for International Cooperation in Research and Higher Education (STINT, project IG2011-2047), the Swedish Research Council (project 621-2011-6258) and the Spanish Government (project MAT2012-38438-C03, co-funded by the EU through European Regional Development Funds). 


\section{Acknowledgements}

The authors would like to acknowledge Johanna Unosson for her help with helium pychnometry measurements.

\section{References}

1. Zhang J, Liu W, Schnitzler V, Tancret F, Bouler J-M. Calcium phosphate cements for bone substitution: Chemistry, handling and mechanical properties. Acta Biomater. 2014;10(3):1035-1049.

2. Unosson J. Physical properties of acidic calcium phosphate cements. PhD Thesis, Uppsala University, Sweden, 2014.

3. Grover LM, Knowles JC, Fleming GJP, Barralet JE. In vitro ageing of brushite calcium phosphate cement. Biomaterials. 2003;24(23):4133-4141.

4. Tamimi F, Torres J, Lopez-Cabarcos E, Bassett DC, Habibovic P, Luceron E, et al. Minimally invasive maxillofacial vertical bone augmentation using brushite based cements. Biomaterials. 2009;30(2):208-216.

5. Lapczyna H, Galea L, Wüst S, Bohner M, Jerban S, Sweedy A, et al. Effect of grain size and microporosity on the in vivo behaviour of $\beta$-tricalcium phosphate scaffolds. Eur Cells Mater. 2014;28:299-319.

6. Espanol M, Perez RA, Montufar EB, Marichal C, Sacco A, Ginebra MP. Intrinsic porosity of calcium phosphate cements and its significance for drug delivery and tissue engineering applications. Acta Biomater. 2009 Sep $1 ; 5: 2752-2762$.

7. Engstrand Unosson J, Persson C, Engqvist H. An evaluation of methods to determine the porosity of calcium phosphate cements. J Biomed Mater Res B. 2015;103:62-71.

8. van Lenthe GH, Hagenmüller H, Bohner M, Hollister SJ, Meinel L, Müller R. Nondestructive micro-computed tomography for biological imaging and quantification of scaffold-bone interaction in vivo. Biomaterials. 2007;28(15):2479-2490.

9. Bohner M, Lemaître J, Ring TA. Effects of sulfate, pyrophosphate, and citrate ions on the physicochemical properties of cements made of $\beta$ tricalcium phosphate-phosphoric acid-water mixtures. J Am Chem Soc. 1996;79(6):1427-1434.

10. Pittet C, Lemaître J. Mechanical characterization of brushite cements: A Mohr circles' approach. J Biomed Mater Res. 2000;53(6):769-780.

11. Gorst NJS, Perrie Y, Gbureck U, Hutton AL, Hofmann MP, Grover LM, et al. Effects of fibre reinforcement on the mechanical properties of brushite 
cement. Acta Biomater. 2006 Jan;2(1):95-102.

12. Barralet JE, Grover LM, Gbureck U. Ionic modification of calcium phosphate cement viscosity. Part II: hypodermic injection and strength improvement of brushite cement. Biomaterials. 2004;25(11):2197-2203.

13. Lamberet S. Durability of ternary binders based on Portland cement, calcium aluminate cement and calcium sulfate. École Polytechnique Fédérale de Lausanne, Switzerland, 2005.

14. Canal C, Pastorino D, Mestres G, Schuler P, Ginebra M-P. Relevance of microstructure for the early antibiotic release of fresh and pre-set calcium phosphate cements. Acta Biomater. 2013;9(9):8403-8412.

15. Ginebra MP, Driessens FCM, Planell JA. Effect of the particle size on the micro and nanostructural features of a calcium phosphate cement: a kinetic analysis. Biomaterials. 2004;25(17):3453-3462.

16. Unosson J, Engqvist H. Development of a resorbable calcium phosphate cement with load bearing capacity. Bioceram Dev Appl. 2014;4(1):074.

17. ASTM. ASTM F 451-08: Standard specification for acrylic bone cement. 2008.

18. Taut T, Kleeberg R, Bergmann J. Seifert Software: The new Seifert Rietveld program BGMN and its application to quantitative phase analysis. Mater Struct. 1998;5(1):57-66.

19. ASTM E177-14: Standard practise for use of the terms precision and bias in ASTM test methods. 2013.

20. Döbelin N. Interlaboratory study on the quantification of calcium phosphate phases by Rietveld refinement. Powder Diffr, 2015.

21. Sudarsanan K, Young RA. Significant precision in crystal structure details: Holly Springs hydroxyapatite. Acta Crystallogr C. B25: 1969;1534-1543.

22. Mathew M, Schroeder LW, Dickens B, Brown WE. The crystal structure of $\alpha-\mathrm{Ca}_{3}\left(\mathrm{PO}_{4}\right)_{2}$. Acta Crystall B-Stru. 1977;33(5):1325-1333.

23. Dickens B, Schroeder LW, Brown WE. Crystallographic studies of the role of $\mathrm{Mg}$ as a stabilizing impurity in $\beta-\mathrm{Ca}_{3}\left(\mathrm{PO}_{4}\right)_{2}$. The crystal structure of pure $\beta-\mathrm{Ca}_{3}\left(\mathrm{PO}_{4}\right)_{2}$. J Solid State Chem. 1974;10(3):232-248.

24. Curry NA, Jones DW. Crystal structure of brushite, calcium hydrogen orthophosphate dihydrate: a neutron-diffraction investigation.J Chem Soc, A. 1971;3725-3729.

25. Boudin S, Grandin A, Borel MM, Leclaire A, Raveau B. Redetermination of the $\beta-\mathrm{Ca}_{2} \mathrm{P}_{2} \mathrm{O}_{7}$ structure. Acta Crystallogr C. 1993;C49:2062-2064.

26. Dickens B, Bowen JS, Brown WE. A refinement of the crystal structure of 
$\mathrm{CaHPO}_{4}$ (synthetic monetite). Acta Crystallogr C. 1971;B28:797-806.

27. Rice RW. Microstructure dependence of mechanical behaviour of ceramics. In: McCrone RK, editor. Treatise on Materials Science and Technology. New York: Academic Press; 1977. p. 199-381.

28. Engstrand J, Persson C, Engqvist H. The effect of composition on mechanical propertiesof brushite cements. J Mech Behav Biomed. 2014;29(c):81-90. 